\section{PREVALENCIA E FATORES ASSOCIADOS AO EXCESSO DE PESO EM UMA POPULAÇÃO URBANADO INTERIOR DO AMAZONAS, BRASIL}

\author{
Excess weight prevalence and associated factors in an urban \\ population from the Amazonas countryside, Brazil
}

\author{
Prevalencia y factores asociados con el exceso de peso de una \\ población urbana del interior de Amazonas, Brasil
}

\section{RESUMO}

Objetivo: Descrever a prevalência do sobrepeso na população adulta residente no município de Tefé, Amazonas, segundo características sociodemográficas, de estilo de vida e de saúde. Métodos: Estudo descritivo, transversal, de base populacional, avaliou, por meio de inquérito domiciliar, 451 indivíduos com idade $\geq 18$ anos, em 2015. Utilizou-se questionário para descritores sociodemográficos (gênero, idade, estado civil, escolaridade, renda familiar), estilo de vida (tabagismo, etilismo, sedentarismo), saúde (perfil nutricional, obesidade, diabetes mellitus, hipertensão), e risco para sobrepeso e obesidade, com $\alpha=0,05$. Resultados: Encontrou-se $22,7 \%(\mathrm{n}=102)$ da população de Tefé com sobrepeso $\left(\mathrm{IC}_{95 \%}: 20,1-\right.$ 24,6). Contudo, a prevalência do sobrepeso é 1,42 vezes maior nos indivíduos com idade $\geq 36$ anos quando comparada à faixa de 18 a 35 anos $\left(\mathrm{IC}_{95 \%}: 1,00-2,02\right)$. Prevalência maior observada entre hipertensos $\left(1,58 ; \mathrm{IC}_{95 \%}: 1,11-2,25\right)$, os que não consomem pescado com regularidade $\left(8,98 ; \mathrm{IC}_{95 \%}: 6,37-12,68\right)$ e naqueles com circunferência abdominal alterada $\left(3,5 ; \mathrm{IC}_{95 \%}: 2,41-5,07\right)$. A análise mostrou associação do aumento do IMC em função do gênero e da circunferência abdominal. Na análise ajustada para a idade, consumo de pescado e hipertensão, o risco para o sobrepeso foi de 10,07 para indivíduos com circunferência abdominal aumentada $\left(\mathrm{IC}_{95 \%}: 5,01-20,26 ; \mathrm{p}=0,00\right)$ e de 3,94 para os homens $\left(\mathrm{IC}_{95 \%}: 2,04-7,61\right.$; $\mathrm{p}=0,00)$. Conclusão: Os resultados apontam que na população adulta de Tefé, a prevalência do sobrepeso é maior entre os homens com acentuado índice de gordura abdominal.

Descritores: Fator de Risco; Epidemiologia; Sobrepeso.

\section{ABSTRACT}

Objective: To describe the prevalence of overweight in the adult population from the municipality of Tefé, Amazonas, according to sociodemographic, lifestyle and health-related characteristics. Methods: Descriptive cross-sectional population-based study that assessed 451 individuals aged $\geq 18$ years in 2015 using a household survey. A questionnaire was used to assess sociodemographic descriptors (gender, age, marital status, education, household income), lifestyle (smoking, drinking, physical inactivity) and health (nutritional profile, obesity, diabetes mellitus, hypertension) and risk for overweight and obesity, with $\alpha=0.05$. Results: It was found that $22.7 \%(n=102)$ of the population of Tefé is overweight ${ }_{95 \%} \mathrm{CI}$ : 20.1-24.6). However, the prevalence of overweight is 1.42 times higher in individuals aged $\geq 36$ years compared to those aged 18-35 years $\left(_{95 \%} \mathrm{CI}: 1.00-2.02\right)$. Higher prevalence rates were observed among hypertensive individuals (1.58; ${ }_{95 \%}$ CI: 1.11-2.25), those who do not eat fish frequently (8.98; ${ }_{95 \%}$ CI: 6.37-12.68) and those with increased abdominal circumference (3.5; ${ }_{95 \%}$ CI: 2.41-5.07). The analysis demonstrated an association between increased BMI by gender and abdominal circumference. In the analysis adjusted for age, fish consumption and hypertension, the risk for overweight was 10.07 for individuals with increased abdominal circumference $\left.{ }_{95 \%} \mathrm{CI}: 5.01-20.26, p=0.00\right)$ and 3.94 for men $\left.{ }_{95 \%} C I: 2.04-7.61 ; p=0.00\right)$. Conclusion: The results show that the prevalence of overweight in the population of Tefé is higher among men with increased abdominal fat.

Descriptors: Risk Factor; Epidemiology; Overweight.

\section{Artigo Original}

Jéssica Yelle Ferreira Cordeiro ${ }^{(1)}$ Sílvia Regina Sampaio Freitas ${ }^{(1)}$

1) Universidade do Estado do Amazonas UEA - Tefé (AM) - Brasil
Recebido em: 24/06/2016 Revisado em: 17/08/2016 Aceito em: 31/10/2016 


\section{RESUMEN}

Objetivo: Describir la prevalencia de sobrepeso de la población adulta del municipio de Tefé/Amazonas, según las características sociodemográficas, de estilo de vida y de salud. Métodos: Estudio descriptivo, transversal, de base poblacional que evaluó a través de una encuesta domiciliaria 451 individuos con edad $\geq 18$ años, en 2015. Se utilizó un cuestionario para los descriptores sociodemograficos (género, edad, estado civil, escolaridad, renta familiar), el estilo de vida (tabaquismo, etilismo, sedentarismo), la salud (perfil nutricional, obesidad, diabetes mellitus, hipertensión) y el riesgo para el sobrepeso y la obesidad, con $\alpha=0,05$. Resultados: Se encontró el 22,7\% $(n=102)$ de la población de Tefé con sobrepeso $\left(I C_{95 \%}: 20,1-\right.$ 24,6). Sin embargo, la prevalencia del sobrepeso es 1,42 veces mayor en los individuos con edad $\geq 36$ años al comparar a la franja de edad entre 18 y 35 años $\left(I C_{95 \%}\right.$ : 1,00-2,02). Se observó mayor prevalencia entre los hipertensos $\left(1,58 ; I C_{95 \%}: 1,11-2,25\right)$, los que no consumen pescado con regularidad $\left(8,98 ; I C_{95 \%}: 6,37-\right.$ 12,68) y aquellos con alteración de la circunferencia abdominal $\left(3,5 ; I C_{95 \%}: 2,41-5,07\right)$. El análisis mostró la asociación entre el aumento del IMC en función del género y de la circunferencia abdominal. En el análisis ajustado para la edad, el consumo del pescado e hipertensión, el riesgo para el sobrepeso fue de 10,07 para los individuos con la circunferencia abdominal aumentada $\left(I C_{95 \%}: 5,01-20,26 ; p=0,00\right)$ y de 3,94 para los hombres $\left(I C_{95 \%}\right.$ : 2,04-7,61; $p=0,00)$. Conclusión: Los resultados señalan que en la población adulta de Tefé la prevalencia del sobrepeso es mayor para los hombres con elevado indice de grasa abdominal.

Descriptores: Factores de Riesgo; Epidemiología; Sobrepeso.

\section{INTRODUÇÃO}

O excesso de peso (ou sobrepeso) é um nível do estado nutricional caracterizado pelo peso corporal aumentado, derivado do acúmulo de gordura em relação à altura, que pode propiciar risco elevado para o desenvolvimento de diversas doenças ${ }^{(1)}$. A causa fundamental do excesso de peso é o desequilíbrio entre o consumo calórico e o gasto energético, decorrente da transição nutricional e urbanização que aparece de forma crescente ${ }^{(1)}$. A obesidade é o estado mais grave do excesso de peso, sendo caracterizada como uma das doenças que integra o grupo de doenças e agravos não transmissíveis ${ }^{(2)}$. Nas últimas décadas, a obesidade passou a ser um dos maiores problemas de saúde por estar associada a uma série de agravos na saúde, tais como: diabetes mellitus, cardiopatias, dislipidemias, distúrbios psicossociais, apneia do sono, osteoartrites e hipertensão $\operatorname{arterial}^{(2,3)}$.

No Brasil, verifica-se a tendência de aumento da prevalência do sobrepeso e da obesidade como consequência do processo de transição nutricional. Segundo os dados da
Secretaria de Vigilância em Saúde do Ministério da Saúde, em 2006, o índice de indivíduos adultos com sobrepeso era de $43 \%$, enquanto os obesos representavam $13 \%$ da população ${ }^{(4)}$. Assim como no Brasil, estudos realizados na Europa $^{(5)}$, nos Estados Unidos ${ }^{(6)}$ e em países emergentes da América Latina ${ }^{(7)}$ também apontam para um crescimento linear e gradual no número de indivíduos com excesso de peso ou com obesidade.

Atualmente, estes índices chegam a 52,5\%, sendo que $18 \%$ da população é considerada obesa ${ }^{(8)}$. Essa tendência de aumento de prevalência do sobrepeso e obesidade é verificada para todas as regiões brasileiras ${ }^{(8)}$.

A Região Amazônica apresenta uma situação especial na realidade brasileira, pois ao mesmo tempo em que possui grande parte de sua população vivendo isoladamente e com hábitos ribeirinhos mantidos, também possui centros urbanos onde predominam os problemas das grandes cidades $^{(9)}$.

Estudos populacionais de âmbito nacional indicam uma tendência de aumento no número de casos de sobrepeso e obesidade nas capitais da Região Amazônica ${ }^{(10)}$. Investigações recentes têm associado estes resultados às mudanças no estilo de vida em virtude de projetos de infraestrutura ${ }^{(11)}$, processos migratórios ${ }^{(12)}$ e mudanças no comportamento alimentar ${ }^{(13)}$. Logo, os impactos socioambientais oriundos de tais fatores sobre o estilo de vida podem ser suficientemente importantes a ponto de alterar o perfil de morbimortalidade das populações residentes na Região Amazônica ${ }^{(11,12)}$

Mesmo com essas especificidades, é reconhecido que o sobrepeso/obesidade é um agravo crônico não transmissível que tem afetado vários segmentos populacionais ${ }^{(14)}$. Portanto, estudos sistemáticos sobre a situação de saúde das populações amazônicas e seus principais condicionantes permitirão comparações com avaliações futuras e a possível detecção das fragilidades do sistema de saúde, além de ampliar o entendimento da magnitude e as tendências históricas dos riscos de morbidade e mortalidade nesses grupos populacionais ${ }^{(11,12)}$.

A literatura sobre as condições sociais, econômicas, demográficas e de saúde dos diferentes grupos populacionais da Região Amazônica é restrita às populações urbanas das capitais, como Manaus ${ }^{(10)}$, Belém ${ }^{(10)}$ e Rio Branco ${ }^{(10,12)}$. Assim, é também necessário averiguar os fatores que contribuem para o aumento do peso corpóreo em cidades de pequeno e médio porte, considerando as discrepâncias das condições de vida diferenciadas entre as cidades brasileiras.

Portanto, diante deste fato, o presente estudo objetivou descrever a prevalência do sobrepeso na população adulta residente no município de Tefé, Amazonas, segundo características sociodemográficas, de estilo de vida e de saúde. 


\section{MÉTODOS}

Trata-se de estudo observacional, descritivo e transversal, de base populacional, realizado no período de março a novembro de 2015, por meio de inquérito domiciliar. A abordagem metodológica envolveu o uso de questionários padronizados e estruturados seguido de análise antropométrica para uma amostra da população adulta (idade superior a 18 anos) residente no município de Tefé, Amazonas, Brasil.

Tefé está localizada na região do Médio Solimões e ocupa uma área territorial de $23.704 \mathrm{Km}^{2(15)}$. Segundo o Censo de 2010, a população de Tefé possui 61.453 habitantes, sendo 31.492 habitantes do sexo masculino e 29.907 do feminino ${ }^{(16)}$. Aproximadamente $81,48 \%$ da população residem na área urbana do município e 18,52\% na área rural ${ }^{(16)}$.

O tamanho da amostra foi estimado com o objetivo de assegurar representatividade para o evento em estudo. O cálculo da amostra utilizou as informações do Censo Demográfico referente à população residente da área urbana relatada pelo Instituto Brasileiro de Geografia e Estatística (IBGE) ${ }^{(16)}$ no ano de 2010 para o município de Tefé, perfazendo um total de 61.453 adultos, tendo sido estabelecido o número de 380 indivíduos a serem estudados. Utilizou-se, para calcular a amostra, o programa estatístico Epi-Info (versão 3.5.1), com intervalo de confiança de $95 \%$, proporção de $50 \%$ e erro de estimação de 5\%. Com acréscimo de $20 \%$ para possíveis perdas e/ou recusas, o tamanho total da amostra foi de 451 adultos.

A seleção da amostra teve como unidade de referência as Áreas de Expansão Demográfica (EAD), segundo o IBGE $^{(16)}$, totalizando 18 EAD em Tefé. De cada setor, selecionou-se uma amostra aleatória simples, proporcional ao número de indivíduos residentes em cada um desses setores, tendo em vista o tamanho da amostra. Devido à proporção considerada e para melhor distribuição para efeitos de vizinhança, foi sorteado um domicílio e saltados cinco. No caso do domicílio estar vazio, escolheu-se a casa imediatamente seguinte. Compuseram os critérios de exclusão: gestantes, mães de crianças menores de seis meses e pessoas com limitações físicas e/ou mentais que impossibilitassem a obtenção dos dados.

A investigação epidemiológica dos descritores demográficos, socioeconômicos, de estilo de vida e de saúde ocorreu por meio de questionário elaborado pelos pesquisadores especificamente para este estudo. Uma equipe multidisciplinar, formada por uma técnica em enfermagem e um biólogo (anotador), ficou responsável por realizar as entrevistas, aplicar os questionários e avaliar os descritores da saúde. As entrevistas, previamente agendadas com um dos responsáveis pelo domicílio conforme a disponibilidade e conveniência do entrevistado, tiveram duração média de 35 minutos e ocorreram na residência do participante. No momento da entrevista, o biólogo lia as perguntas e anotava as respostas em uma planilha de dados gravada no dispositivo móvel exclusivo deste estudo (tablet do modelo WorkTab Q10; Philco). Em seguida, a técnica de enfermagem realizava a avaliação dos descritores de saúde. O entrevistado permanecia sentado durante a avaliação do comportamento alimentar, medição da glicose e aferição da pressão arterial, e em pé para as verificações de peso, altura e circunferência abdominal. No decorrer desta etapa, o biólogo permaneceu ao lado da avaliadora a fim de anotar os resultados na planilha de dados. Ao final do dia, as informações contidas no dispositivo móvel eram repassadas para um HD externo (modelo HD-EG5; Sony).

As variáveis demográficas e socioeconômicas estudadas incluíam: sexo, idade, estado civil (solteiro, casado/unido, divorciado/separado), nível de escolaridade (ensino fundamental completo/incompleto, ensino médio completo/ incompleto, ensino superior completo/incompleto) e renda familiar mensal em salários mínimos.

Quanto ao estilo de vida, investigou-se: o tabagismo, categorizado em não fumante ou fumante ativo $(\geq 1$ maço por semana); o consumo de bebidas alcoólicas em relação aos 30 dias anteriores à entrevista; e a atividade física, mensurada no tempo de lazer em minutos por semana.

As variáveis de saúde abordaram a avaliação dos hábitos dietéticos, a determinação do índice de massa corporal (IMC), a medição da circunferência abdominal (CA), a aferição da pressão arterial e a determinação da glicose.

A avaliação dos hábitos dietéticos dos entrevistados fundamentou-se em informações sobre consumo e frequência de consumo de: carne (carne vermelha, carne branca, e pescado), refrigerantes e sucos artificiais. $\mathrm{O}$ hábito de fazer as principais refeições (café da manhã, almoço e jantar) em casa ou em padaria/restaurante também fez parte da análise do perfil alimentar. Para nortear a avaliação do perfil nutricional, utilizou-se como base o questionário da Pesquisa Nacional de Saúde ${ }^{(17)}$. Os parâmetros da avaliação alimentar foram categorizados em: "consumidor de carne ou ave com gordura" (ingesta de carne vermelha ou de frango, independente da quantidade ou frequência semanal, com gordura visível ou pele respectivamente) e "não consumidor de gordura ou pele" (atribuído aos que informaram retirar e descartar a gordura da carne vermelha ou a pele da carne de frango/galinha); "consumidor frequente de pescado" (aqueles que afirmaram consumir pescado duas ou mais vezes por semana) e "consumidor não frequente" (aos que indicaram consumir pescado com frequência inferior a duas vezes por semana); "consumidor regular de refrigerantes/ sucos artificiais" (quando a ingesta de bebidas artificiais 
atingiu cinco dias ou mais por semana) e "consumidor não regular" (atribuído àqueles que afirmaram frequência inferior a cinco vezes por semana); "refeição em casa" (indivíduos que referiram realizar as principais refeições café da manhã, almoço e jantar - em casa) e "refeição na rua" (aos que informaram fazer as principais refeições em estabelecimentos comerciais).

Pelas medidas de peso e altura obtidas na coleta de dados, calculou-se o IMC de cada indivíduo através da divisão do peso (massa corporal em quilogramas) pelo quadrado da altura (em metros). A caracterização do estado nutricional da população de estudo fundamentou-se nos pontos de corte para o IMC, como preconizados pela Organização Mundial de Saúde (OMS) ${ }^{(18)}$, classificando os indivíduos como normal $\left(<25 \mathrm{~kg} / \mathrm{m}^{2}\right)$ e sobrepeso $(\geq 25 \mathrm{~kg} /$ $\mathrm{m}^{2}$ ). Os entrevistadores procederam a aferição do peso do entrevistado com o auxílio de balança portátil (modelo BF572 Body Fat, Tanita Corp., capacidade de 130kg com intervalos de $100 \mathrm{~g}$ ), e medição da altura pelo estadiômetro portátil graduado em milímetros. A aferição do peso ocorreu com os indivíduos com os pés descalços e trajando roupas leves.

A medição da circunferência abdominal, realizada com treina antropométrica (modelo R88, WISO), considerou a metade da distância entre a crista ilíaca superior anterior e a última costela, no final da expiração. Com base nos critérios da OMS para classificação de normalidade da $\mathrm{CA}^{(18)}$, homens com até $102 \mathrm{~cm}$ e mulheres com $88 \mathrm{~cm}$ de circunferência abdominal foram considerados normais. Valores superiores indicaram obesidade abdominal.

A aferição da pressão arterial ocorreu em triplicada ${ }^{(19)}$ e com aparelho semiautomático (modelo HEM 711AC, OMRON), considerando-se para análise a média das três medições. No momento da avaliação pressórica, o participante encontrava-se sentado, após cinco minutos de repouso e com abstinência, de 30 minutos, de café ou tabaco. Foram considerados hipertensos os que apresentaram pressão arterial sistólica (PAS) $\geq 140 \mathrm{mmHg}$ e/ou pressão arterial diastólica $(\mathrm{PAD}) \geq 90 \mathrm{mmHg}^{(19)}$ e aqueles que relataram uso, ainda que irregular, de alguma medicação anti-hipertensiva $^{(19)}$.

O diagnóstico de diabetes mellitus (DM) baseou-se no histórico, uso de medicamentos hipoglicemiantes e medida de glicose sérica superior a $100 \mathrm{mg} / \mathrm{dL}^{(20)}$. A medição dos níveis plasmáticos ocorreu por meio de kit comercial (AccuChek Active, ROCHE), na residência do entrevistado.

As análises estatísticas, conduzidas pelo programa SPSS (versão 20.0), incluíram: 1) análise descritiva, evidenciando as frequências absolutas, relativas, valores de média e desvio padrão, e as razões de prevalência (RP) com seus respectivos intervalos de confiança $\left.\left(\mathrm{IC}_{95 \%}\right) ; 2\right)$ análise comparativa dos parâmetros demográficos, socioeconômicos, de estilo de vida e de saúde, para averiguar diferenças e similaridades entre os grupos de indivíduos magros $\left(<25 \mathrm{~kg} / \mathrm{m}^{2}\right)$ e com sobrepeso $\left(\geq 25 \mathrm{~kg} / \mathrm{m}^{2}\right)$, com uso do teste de $t$-Student para variáveis contínuas e o teste de Qui-quadrado para variáveis qualitativas; 3) regressão logística múltipla, para estimar o efeito das variáveis explicativas sobre a variação do peso corpóreo.

Esta pesquisa respeitou os padrões éticos e científicos estabelecidos pela Resolução $\mathrm{n}^{\circ}$. 466, de 12 de dezembro de 2012, do Conselho Nacional de Saúde - Ministério da Saúde, sobre pesquisa envolvendo seres humanos; tendo sido aprovada pelo Comitê de Ética em Pesquisa da Universidade do Estado do Amazonas, mediante o parecer $\mathrm{n}^{\circ}$. 407.080, de 26 de setembro de 2013. Os entrevistados assinaram o Termo de Consentimento Livre e Esclarecido para participação na pesquisa.

\section{RESULTADOS}

Os resultados da análise descritiva estão sumarizados na Tabela I. A amostra de 451 adultos da população de Tefé, AM foi constituída de 41,7\% ( $\mathrm{n}=188$ ) homens, idade média de $38,42 \pm 14,69$ anos, e $58,3 \%(n=263)$ mulheres com média de 38,20 $\pm 14,42$ anos. A maioria dos entrevistados $(51,8 \%, \mathrm{n}=231)$ tinha mais de 36 anos de idade e ensino superior $(42,9 \%, \mathrm{n}=189)$. Quanto à renda familiar, 49,5\% $(n=218)$ dos entrevistados informaram rendimento mensal entre 3 e 5 salários mínimos. Dos participantes, $47 \%(n=211)$ são solteiros, enquanto $45,4 \%(n=204)$ são casados. A proporção de divorciados, separados ou viúvos atingiu 7,6\% $(\mathrm{n}=34)$ da amostra. Quanto ao estilo de vida, apenas $6,2 \%(\mathrm{n}=28)$ declarou ser fumante. Contudo, $34,8 \%(\mathrm{n}=157)$ dos entrevistados afirmaram ter o hábito de consumir bebidas alcoólicas e $60,1 \%(n=268)$ relataram não praticar atividades físicas.

Com relação às variáveis de saúde, verificaram-se os valores médios de $126,04 \pm 18,34 \mathrm{mmHg}$ de pressão arterial sistólica, 79,83 $\pm 13,14 \mathrm{mmHg}$ de pressão arterial diastólica, $93,77 \pm 29,31 \mathrm{mg} / \mathrm{dL}$ de glicemia em jejum, $21,89 \pm 4,19 \mathrm{~kg} /$ $\mathrm{m}^{2}$ de índice de massa corporal, e $91,29 \pm 14,94 \mathrm{~cm}$ de CA em mulheres e $91,55 \pm 15,12 \mathrm{~cm}$ de CA em homens.

A análise do perfil alimentar dos moradores de Tefé mostrou que $26,9 \%(n=121)$ consomem a gordura da carne da carne vermelha e/ou a pele da carne branca, enquanto $71,4 \%(n=322)$ ingerem pescado com frequência superior a duas vezes por semana. Apenas $12 \%(\mathrm{n}=52)$ realizam as principais refeições em restaurantes ou padarias e 15,4\% $(n=73)$ afirmaram consumir com regularidade sucos artificiais ou refrigerantes.

A análise bivariada (Tabela II) evidenciou que a prevalência estimada do sobrepeso é de $22,7 \%\left(\mathrm{IC}_{95 \%}: 20,1\right.$ 
Tabela I - Perfil demográfico, de estilo de vida e saúde da população adulta investigada. Tefé, AM, 2015.

\begin{tabular}{|c|c|}
\hline \multirow{2}{*}{ Variáveis } & \multirow{2}{*}{$\begin{array}{c}\text { Análise descritiva } \\
\mathbf{n}(\%)\end{array}$} \\
\hline & \\
\hline \multicolumn{2}{|l|}{ Características Socioeconômicas } \\
\hline \multicolumn{2}{|l|}{ Sexo } \\
\hline Feminino & $263(58,3 \%)$ \\
\hline Masculino & $188(41,7 \%)$ \\
\hline \multicolumn{2}{|l|}{ Idade, anos } \\
\hline Até 35 anos & $215(48,2 \%)$ \\
\hline A partir de 36 anos & $231(51,8 \%)$ \\
\hline \multicolumn{2}{|l|}{ Nível de Escolaridade } \\
\hline Ensino Fundamental & $142(32,2 \%)$ \\
\hline Ensino Médio & $110(24,9 \%)$ \\
\hline Ensino Superior & $189(42,9 \%)$ \\
\hline \multicolumn{2}{|l|}{ Renda Familiar, salário mínimo } \\
\hline Até 2 & $188(42,7 \%)$ \\
\hline De 3 a 5 & $218(49,5 \%)$ \\
\hline Acima de 5 & $34(7,7 \%)$ \\
\hline \multicolumn{2}{|l|}{ Estado Civil } \\
\hline Solteiro & $211(47,0 \%)$ \\
\hline Casado/União Estável & $204(45,4 \%)$ \\
\hline Viúvo/Divorciado & $34(7,6 \%)$ \\
\hline \multicolumn{2}{|l|}{ Características de Estilo de Vida } \\
\hline Tabagismo & $28(6,2 \%)$ \\
\hline Consumo de bebida alcoólica & $157(34,8 \%)$ \\
\hline Sedentarismo & $268(60,1 \%)$ \\
\hline \multicolumn{2}{|l|}{ Características de Saúde (qualitativas) } \\
\hline Consumidor de carne ou ave com gordura & $121(26,9 \%)$ \\
\hline Consumo de pescado & $322(71,4 \%)$ \\
\hline Consumidor regular de refrigerante/suco artificial & $73(15,4 \%)$ \\
\hline Realiza as refeições na rua & $52(12 \%)$ \\
\hline Características de Saúde (numéricas) & Média \pm DP \\
\hline \multicolumn{2}{|l|}{ Circunferência Abdominal $(\mathrm{cm})$} \\
\hline Mulheres & $91,29 \pm 14,94$ \\
\hline Homens & $91,55 \pm 15,12$ \\
\hline Pressão Arterial Sistólica, mmHg & $126,04 \pm 18,34$ \\
\hline Pressão Arterial Diastólica, mmHg & $79,83 \pm 13,14$ \\
\hline Glicemia de jejum, mg/dL & $93,77 \pm 29,31$ \\
\hline
\end{tabular}

n: valor absoluto; DP: desvio padrão; cm: centímetro; mmHg: milímetro de mercúrio; mg/dL: miligrama de glicose por decilitro de sangue.

- 24,6) da população adulta, não apresentando diferença significativa entre mulheres $(20,3 \%)$ e homens $(26,1 \%)$. $\mathrm{Na}$ análise por faixa etária, constatou-se que a prevalência para o sobrepeso é 1,42 vezes maior nos indivíduos com idade $\geq 36$ anos quando comparada com a faixa de 18 a
35 anos $\left(\mathrm{IC}_{950}: 1,00-2,02\right)$. As variáveis de estado civil (solteiro: 1,05 [ $\mathrm{IC}_{950}: 0,48$ - 2,26]; casado/união estável: $1,48\left[\mathrm{IC}_{95 \%}: 0,72\right.$ - 3,06]), renda familiar (de 3-5 salários mínimos: $1,25\left[\mathrm{IC}_{95 \%}: 0,86\right.$ - 1,80; acima de 5 salários mínimos: $1,32\left[\mathrm{IC}_{95 \%}: 0,69\right.$ - 2,51]) e nível de escolaridade 
Tabela II - Prevalência do sobrepeso/obesidade, razão de prevalência e intervalo de confiança, segundo características socioeconômicas da população adulta investigada. Tefé, AM, 2015.

\begin{tabular}{|c|c|c|c|c|}
\hline \multirow{2}{*}{ Variáveis } & \multicolumn{2}{|c|}{ Grupos } & \multirow{2}{*}{ Prevalência } & \multirow{2}{*}{ RP [IC95\%] } \\
\hline & IMC $<25$ & IMC $\geq 25$ & & \\
\hline \multicolumn{5}{|l|}{ Gênero } \\
\hline Feminino & 204 & 52 & $20,3 \%$ & 1,00 \\
\hline Masculino & 136 & 48 & $26,1 \%$ & $1,28[0,91-1,81]$ \\
\hline \multicolumn{5}{|l|}{ Idade (anos) } \\
\hline Até 35 anos & 170 & 39 & $18,7 \%$ & 1,00 \\
\hline A partir de 36 anos & 166 & 60 & $26,5 \%$ & $1,42[1,00-2,02]$ \\
\hline \multicolumn{5}{|l|}{ Nível de Escolaridade } \\
\hline Ensino Superior & 145 & 40 & $21,6 \%$ & 1,00 \\
\hline Ensino Médio & 86 & 22 & $20,3 \%$ & $0,94[1,49-0,59]$ \\
\hline Ensino Fundamental & 102 & 35 & $25,5 \%$ & $1,18[0,79-1,76]$ \\
\hline \multicolumn{5}{|l|}{ Renda Familiar } \\
\hline Até 2 & 147 & 37 & $20,1 \%$ & 1,00 \\
\hline De 3 a 5 & 158 & 53 & $25,1 \%$ & $1,25[0,86-1,80]$ \\
\hline Acima de 5 & 25 & 09 & $26,4 \%$ & $1,32[0,69-2,51]$ \\
\hline \multicolumn{5}{|l|}{ Estado Civil } \\
\hline Solteiro & 166 & 39 & $19,0 \%$ & $1,05[0,48-2,26]$ \\
\hline Casado/Outros & 146 & 54 & $27,0 \%$ & $1,48[0,72-3,06]$ \\
\hline Viúvo/Divorciado & 27 & 06 & $18,1 \%$ & 1,00 \\
\hline
\end{tabular}

RP: Razão de Prevalência; IC 95\%: Intervalo de Confiança; IMC $<25$ : Índice de Massa Corporal inferior a 25 kg/m²; IMC $\geq 25$ : Índice de Massa Corporal igual ou superior a $25 \mathrm{~kg} / \mathrm{m}^{2}$.

(ensino médio: 0,94 [ $\mathrm{IC}_{95 \%}: 1,49$ - 0,59]; ensino fundamental: $\left.1,18\left[\mathrm{IC}_{95 \%}: 0,79-1,76\right]\right)$ não apresentaram associação com o sobrepeso.

Com relação às variáveis de saúde (Tabela III), a prevalência do excesso de peso está 1,58 vezes mais elevada entre os indivíduos hipertensos quando comparado aos normotensos $\left[\mathrm{IC}_{95 \%}: 1,11\right.$ - 2,25]. Entre os que não consomem pescado com regularidade, o índice de sobrepeso aumenta para 8,98 [ $\left.\mathrm{IC}_{95} \%: 6,37-12,68\right]$. Além disso, verificou-se que a prevalência da obesidade abdominal, medida pela circunferência abdominal, é 3,5 vezes maior no grupo de indivíduos com excesso de peso $\left(\mathrm{IC}_{95 \%}: 2,41\right.$ - 5,07). O perfil glicêmico $\left(0,98\left[\mathrm{IC}_{95 \%}: 1,49-0,65\right]\right)$, o padrão alimentar (consumo de carne vermelha ou branca com gordura: 1,23 $\left[\mathrm{IC}_{95 \%}: 0,81-1,87\right]$; consumo regular de refrigerantes ou sucos artificiais: $1,37\left[\mathrm{IC}_{95 \%}: 0,90-2,08\right]$; alimentar-se em estabelecimentos comerciais: $\left.1,01 \quad\left[\mathrm{IC}_{95 \%}: 0,58-1,77\right]\right)$ e as variáveis de estilo de vida, que são o tabagismo $(0,97$ $\left[\mathrm{IC}_{95 \%}: 2,00\right.$ - 0,47]), o sedentarismo $\left(1,35\left[\mathrm{IC}_{95 \%}: 0,96\right.\right.$ $1,91])$ e o consumo de bebidas alcoólicas $\left(1,12\right.$ [IC $_{95 \%}: 0,79$ - 1,60]), não apresentaram associação com a prevalência do excesso de peso no presente estudo.
A análise de regressão logística múltipla mostrou associação do aumento do IMC em função do gênero e da circunferência abdominal. A análise multivariada para o excesso de peso, ajustada para a idade, consumo de pescado, hipertensão e diabetes, também determinou a sua prevalência de 10,07 para indivíduos com circunferência abdominal aumentada $\left(\mathrm{IC}_{95} \%: 5,01-20,26 ; \mathrm{p}=0,00\right)$ e de 3,94 para os homens $\left(\mathrm{IC}_{95} \%: 2,04-7,61 ; \mathrm{p}=0,00\right)$.

\section{DISCUSSÃO}

Este estudo visou descrever a prevalência do excesso de peso de acordo com descritores sociodemográficos, de estilo de vida e de saúde em uma amostra representativa da população adulta de Tefé/AM. A identificação de descritores de risco para o excesso de peso permite ações de saúde pública, conforme condições de vida diferenciadas da região estudada, direcionadas à redução da morbimortalidade e melhor qualidade de vida da população.

Nas últimas décadas, o excesso de peso e a obesidade passaram a ser um dos maiores problemas de saúde da humanidade devido ao aumento de suas prevalências em 
Tabela III - Prevalência do sobrepeso/obesidade, razão de prevalência e intervalo de confiança, segundo as características de saúde e de estilo de vida na população adulta investigada. Tefé, AM, 2015.

\begin{tabular}{|c|c|c|c|c|}
\hline \multirow{2}{*}{ Variáveis } & \multicolumn{2}{|c|}{ Amostras } & \multirow{2}{*}{ Prevalência } & \multirow{2}{*}{ RP [IC95\%] } \\
\hline & IMC $<25$ & IMC $\geq 25$ & & \\
\hline \multicolumn{5}{|c|}{ Consumo de carne/ave com gordura } \\
\hline Não & 251 & 77 & $23,5 \%$ & 1,00 \\
\hline Sim & 98 & 23 & $19,0 \%$ & $1,23[0,81-1,87]$ \\
\hline \multicolumn{5}{|c|}{ Consumo frequente de pescado } \\
\hline Sim & 300 & 22 & $6,8 \%$ & 1,00 \\
\hline Não & 49 & 78 & $61,4 \%$ & $8,98[6,37-12,68]$ \\
\hline \multicolumn{5}{|c|}{ Consumo regular de refrigerantes ou sucos artificiais } \\
\hline Não & 298 & 80 & $21,2 \%$ & 1,00 \\
\hline Sim & 49 & 20 & $29,0 \%$ & $1,37[0,90-2,08]$ \\
\hline \multicolumn{5}{|c|}{ Local das Refeições } \\
\hline Em casa & 300 & 82 & $21,5 \%$ & 1,00 \\
\hline $\mathrm{Na}$ rua & 41 & 11 & $21,1 \%$ & $1,01[0,58-1,77]$ \\
\hline \multicolumn{5}{|l|}{ Perfil Pressórico } \\
\hline Normotenso & 200 & 56 & $21,8 \%$ & 1,00 \\
\hline Hipertenso & 140 & 44 & $23,9 \%$ & $1,58[1,11-2,25]$ \\
\hline \multicolumn{5}{|l|}{ Perfil Glicêmico } \\
\hline Até $100 \mathrm{mg} / \mathrm{dL}$ & 216 & 62 & $22,3 \%$ & 1,00 \\
\hline$\geq 100 \mathrm{mg} / \mathrm{dL}$ & 85 & 24 & $22,2 \%$ & $0,98[1,49-0,65]$ \\
\hline \multicolumn{5}{|l|}{$\mathrm{CA}(\mathrm{cm})$} \\
\hline Normal $^{1}$ & 218 & 26 & $10,6 \%$ & 1,00 \\
\hline Aumentada ${ }^{2}$ & 121 & 72 & $37,3 \%$ & $3,50[2,41-5,07]$ \\
\hline \multicolumn{5}{|l|}{ Sedentarismo } \\
\hline Ativo & 128 & 48 & $27,7 \%$ & $1,35[0,96-1,91]$ \\
\hline Sedentário & 207 & 52 & $20,0 \%$ & 1,00 \\
\hline \multicolumn{5}{|l|}{ Tabagismo } \\
\hline Não & 317 & 94 & $21,3 \%$ & 1,00 \\
\hline Sim & 21 & 06 & $22,2 \%$ & $0,97[2,00-0,47]$ \\
\hline \multicolumn{5}{|l|}{ Etilismo } \\
\hline Não & 223 & 62 & $21,7 \%$ & 1,00 \\
\hline Sim & 117 & 38 & $24,5 \%$ & $1,12[0,79-1,60]$ \\
\hline
\end{tabular}

RP: Razão de Prevalência; IC 95\%: Intervalo de Confiança; IMC $<25$ : Índice de Massa Corporal inferior a 25 kg/m²; IMC $\geq 25$ : Índice de Massa Corporal igual ou superior a $25 \mathrm{~kg} / \mathrm{m}^{2} ;$ CA: circunferência abdominal; 1 . Mulher $<88 \mathrm{~cm}$ e Homem $<102 \mathrm{~cm} ; 2$. Mulher $\geq 88 \mathrm{~cm}$ e Homem $\geq 102 \mathrm{~cm}$.

todo o mundo $\mathrm{o}^{(3,5-7)}$. O excesso de peso tem aumentado de forma gradativa em todas as faixas etárias, desde a infância ${ }^{(21)}$ até a idade adulta ${ }^{(14)}$ e a sua ocorrência está associada a uma série de doenças crônico-degenerativas ${ }^{(14)}$. Portanto, faz-se necessária a realização sistemática de investigações epidemiológicas a fim de averiguar associações entre fatores que influenciam o desenvolvimento do excesso de peso/obesidade na população brasileira, principalmente em cidades do interior do país não incluídas na pesquisa da VIGITEL ${ }^{(10)}$.

Os resultados da presente investigação descritiva mostraram que $22,7 \%$ dos adultos residentes de Tefé estão com excesso de peso. Na comparação com outras cidades brasileiras, a prevalência do índice de massa corporal $(\geq$ $25 \mathrm{~kg} / \mathrm{m}^{2}$ ) em Tefé é considerada baixa. Em todas as regiões do país, parcelas significativas da população adulta apresentam excesso de peso e/ou obesidade ${ }^{(8)}$, até mesmo nas capitais da Região Norte ${ }^{(10)}$. Segundo dados do VIGITEL Brasil 2006-2012 $2^{(10)}$, realizado por meio de entrevistas telefônicas, a prevalência de excesso de peso em Rio Branco/AC atingiu $31 \%$ da população adulta, enquanto em Manaus/AM e Belém/PA este índice chegou a, respectivamente, 42,2\% e $46,4 \%$. Estudos de prevalência realizados nas principais capitais mundiais também evidenciaram índices elevados (acima de $35 \%$ ) de casos de excesso de peso e obesidade ${ }^{(22)}$. $\mathrm{O}$ aumento da prevalência de obesidade ocorre tanto em países desenvolvidos ${ }^{(5-6)}$ como em países emergentes ${ }^{(7)}$. O excesso de peso e a obesidade são importantes preditores de risco para doenças crônicas, já que o acúmulo de gordura corporal condiciona a uma série de disfunções endócrinas e metabólicas ${ }^{(1)}$. Ademais, o ganho de peso colabora com aproximadamente $58 \%$ dos casos de diabetes, $21 \%$ das 
cardiopatias isquêmicas e entre $8 \%$ a $42 \%$ de certos tipos de câncer ${ }^{(23)}$.

Ao investigar os motivos para o baixo índice de indivíduos com sobrepeso no presente estudo, verificouse que a dieta da população de Tefé ainda conta com importante participação de alimentos reconhecidos como marcadores de alimentação saudável. A maioria dos participantes do estudo relatou o consumo regular de peixe, enquanto uma minoria informou consumir carne vermelha ou branca com gordura. Também se observou um baixo consumo de refrigerantes e/ou sucos artificiais. A elevada frequência dos indicadores de alimentação saudável apresentada neste estudo está em consonância com os Dados da Pesquisa Nacional de Saúde (PNS) (24). De acordo com a PNS 2013, 77,2\% dos amazonenses consomem diariamente pescado; sendo a ingesta regular de peixe nos domicílios do Amazonas dez vezes superior quando comparada às demais regiões brasileiras ${ }^{(24)}$. É importante ressaltar também que durante o período das $12 \mathrm{~h}$ às $14 \mathrm{~h}, 90 \%$ dos estabelecimentos comerciais do município investigado no atual estudo encontram-se fechados para o almoço (comunicação pessoal). Esta cultura regional permite que os trabalhadores retornem às suas residências, local onde realizam sua refeição. Portanto, o padrão alimentar decorrente do consumo de alimentos in natura ${ }^{(9)}$ e minimamente processados contribui positivamente para a manutenção do índice de massa corporal dentro dos limites da normalidade ${ }^{(22,25)}$. Assim como verificado em Tefé/ $\mathrm{AM}$, algumas populações asiáticas apresentam índices de sobrepeso e obesidade inferiores a $25 \%{ }^{(22)}$. Tal fenômeno foi associado a uma dieta alimentar rica em pescado e grãos integrais ${ }^{(22)}$.

A alimentação e a nutrição adequadas constituem-se em requisitos básicos para a promoção e a proteção da saúde ${ }^{(26)}$. A Organização Mundial de Saúde aponta para a necessidade de melhoria nos padrões mundiais de alimentação para prevenção, em especial, de doenças crônicas não transmissíveis ${ }^{(26)}$. No Brasil, a alimentação inadequada, a hipertensão arterial e o consumo elevado de álcool compõem os três fatores de risco que mais contribuem para a carga de doenças no país ${ }^{(26)}$. Para a prevenção de doenças cardiovasculares, a OMS recomenda o consumo de peixe na frequência de uma a duas vezes por semana ${ }^{(22,26)}$, assim como verificado na atual pesquisa para a população de Tefé.

A idade foi o segundo preditor do excesso do peso entre os residentes analisados de Tefé. Os resultados do presente estudo mostraram tendência linear significante $(p<0,05)$, com aumento da prevalência de excesso de peso à medida que aumenta a faixa de idade em ambos os sexos. Entre os jovens com idade entre 18 e 35 anos, a prevalência do sobrepeso atingiu $48,2 \%$, enquanto que, entre aqueles com idade superior a 35 anos, o índice de sobrepeso sobe para
51,8\%. Resultados semelhantes em relação à associação do excesso de peso com a idade também foram observados em outros estudos de mesmo delineamento em populações brasileiras $^{(12,27)}$ e mundiais ${ }^{(28)}$. De fato, com a idade, a obesidade apresenta tendência de aumento, proveniente da redução nos níveis de atividade física e diminuição do metabolismo de repouso ${ }^{(23)}$. Tais fatores contribuem para a diminuição do gasto energético, favorecendo assim o ganho de peso corpóreo ${ }^{(23)}$.

No presente estudo, constatou-se que $37,5 \%$ dos indivíduos com circunferência abdominal aumentada possuíam $I M C \geq 25 \mathrm{~kg} / \mathrm{m}^{2}$. Estudos populacionais realizados no Brasil $^{(27)}$ e em países da Europa ${ }^{(5)}$ e Estados Unidos ${ }^{(6)}$ obtiveram prevalências similares, de $28,1 \%, 24,9 \%$ e $33 \%$ respectivamente. Hoje, sabe-se que o tecido adiposo tem múltiplas funções importantes na regulação do balanço, tanto energéticas como metabólicas. O maior impacto negativo da gordura visceral tem sido atribuído às propriedades biológicas distintas desse depósito em relação aos depósitos de outros tecidos ${ }^{(1,23)}$. A atividade funcional dos adipócitos localizados na gordura visceral parece estar condicionada à regulação do Sistema-Renina-AngiotensinaAldosterona, demonstrando uma clara associação entre sobrepeso e pressão arterial ${ }^{(1,23)}$. Embora a circunferência abdominal não possa discriminar entre gordura visceral e gordura subcutânea, pesquisas dão suporte à ideia de que indivíduos com circunferência abdominal elevada têm maior probabilidade de ter hipertensão, diabetes, dislipidemia ou síndrome metabólica, acrescentando informação àquela fornecida pelo $\mathrm{IMC}^{(1,23)}$. Em investigações nas quais foram usadas medidas mais precisas de gordura, foi demonstrado que as associações entre gordura intra-abdominal e várias desordens metabólicas começam a ocorrer na infância ${ }^{(23)}$.

Dos indicadores de saúde avaliados no presente estudo, a hipertensão arterial e o baixo consumo de pescado contribuem para o aumento do peso corpóreo. Segundo os resultados do presente estudo, a prevalência do sobrepeso se apresentou 1,58 vezes maior para aqueles com pressão arterial superior a $140 / 90 \mathrm{mmHg}$. A frequência aumentada de hipertensos entre os indivíduos com sobrepeso sugere o risco de comorbidade e está em consonância com achados na literatura ${ }^{(6,13,21,22,29)}$. É fato que a obesidade e o sobrepeso são importantes indicadores de risco para doenças crônicas não transmissíveis, especialmente as doenças cardiovasculares $^{(1)}$, mostrando a importância de se investir em ações de promoção de alimentação saudável e atividade física ${ }^{(4)}$.

Quanto ao efeito do consumo do pescado para a variação do peso corpóreo, observou-se que a baixa ingesta de peixe favorece o aumento da prevalência do sobrepeso em até 8,98 vezes. Portanto, o consumo de pescado duas ou mais vezes por semana contribui para a manutenção do índice 
de massa corporal dentro dos limites da normalidade ${ }^{(22)}$. Esses efeitos são atribuídos às propriedades nutricionais do peixe, uma vez que este alimento é rico em aminoácidos sulfurados e em ácidos graxos insaturados, o que o torna um importante fator protetor contra o acúmulo de calorias e outros processos degenerativos associados a substâncias tóxicas oxidantes presentes no ambiente ${ }^{(9)}$.

$\mathrm{O}$ modelo multivariado de fatores associados com o sobrepeso mostrou, na pesquisa em questão, associação do aumento do IMC em função do gênero e da circunferência abdominal. Na análise ajustada para a idade e hipertensão, o risco para o sobrepeso foi de 10,07 para indivíduos com circunferência abdominal aumentada, e de 3,94 para os homens. Estudos de amplitude nacional realizados em países como Brasil ${ }^{(10)}$, Estados $\operatorname{Unidos}^{(6)}$ e Austrália ${ }^{(22)}$ evidenciaram o aumento do peso corporal em função da variação da circunferência abdominal bem com o gênero masculino. A relação entre gênero, aumento da circunferência abdominal e sobrepeso advém de padrões comportamentais diferenciados verificados entre os homens e as mulheres ${ }^{(10)}$. Hábitos comportamentais, como o consumo de bebidas alcoólicas, o tabagismo, a ingesta de carne com gordura, são alguns dos fatores mais frequentemente relatados por homens e que favorecem o aumento do peso corpóreo ${ }^{(1,3,4,7,22)}$.

O desenho metodológico do presente estudo apresenta limitações que devem ser consideradas na interpretação dos resultados: (1) a população investigada é aquela que tem maior disponibilidade de ser encontrar em casa, por exemplo, aposentados e donas de casa, e (2) o desenho transversal limita a possibilidade de interpretar associações encontradas como derivadas de relações causa-efeito. Contudo, a realização de um censo demográfico reduziu a possibilidade da ocorrência de vieses neste estudo ${ }^{(8)}$.

O presente estudo relatou a prevalência aumentada do sobrepeso entre os homens com acentuado índice de gordura abdominal no município de Tefé, interior do estado do Amazonas. A população de Tefé, distante $674 \mathrm{~km}$ da capital Manaus ${ }^{(16)}$, reside em meio a uma rica biodiversidade, caracterizada por árvores frutíferas, plantas medicinais e vários grupos de animais silvestres, e apresenta hábitos alimentares baseados no consumo de peixe $^{(16)}$. Um total de $71,4 \%$ da população adulta dessa região consome peixe mais de duas vezes na semana e tem acesso relativamente limitado ao açúcar e a outros itens com maior poder calórico. Apesar da observância de indicadores da alimentação saudável ${ }^{(9,26)}, 26,1 \%$ dos homens apresentam sobrepeso. O risco de ganho de peso devido ao gênero masculino associado com o aumento da circunferência abdominal sinaliza para uma mudança gradual no perfil de morbimortalidade no que se refere, por exemplo, a doenças metabólicas e cardiovasculares ${ }^{(11)}$.
Este estudo representa uma contribuição adicional para o conhecimento dos condicionantes de risco para o sobrepeso e obesidade no interior do Brasil, e poderá contribuir para formulação de estratégias de saúde por parte das secretarias de saúde local e regional.

\section{CONCLUSÃO}

$\mathrm{Na}$ população adulta e residente de Tefé, AM, a prevalência estimada do sobrepeso é de $22,7 \%$ da população adulta, sendo maior nos indivíduos com mais idade, hipertensos e entre os homens com acentuado índice de gordura abdominal.

\section{REFERÊNCIAS}

1. Phillips CM. Metabolically healthy obesity across the life course: epidemiology, determinants, and implications. Ann N Y Acad Sci. 2016;1381(1):1-16.

2. Malta DC, Morais OLD Neto, Silva JBD Junior. Apresentação do plano de ações estratégicas para o enfrentamento das doenças crônicas não transmissíveis no Brasil, 2011 a 2022. Epidemiol Serv Saúde. 2011;20(4):425-38.

3. Malik VS, Willett WC, Hu FB. Global obesity: trends, risk factors and policy implications. Nat Rev Endocrinol. 2013;9(1):13-27.

4. Malta DC, Iser BPM, Claro RM, Moura L, Bernal RTI, Nascimento AF, et al. Prevalência de fatores de risco e proteção para doenças crônicas não transmissíveis em adultos: estudo transversal, Brasil, 2011. Epidemiol Serv Saúde. 2013;22(3):423-34.

5. International Association for the Study of Obesity. Adult overweight and obesity in the European Union (EU27) [Internet] London: IASO; 2012 [acesso em 2016 Out 15]. Disponível em: http://www.iaso.org/ resources/world-map-obesity/

6. Imes CC, Burke LE. The obesity epidemic: the USA as a cautionary tale for the rest of the world. Curr Epidemiol Rep. 2014;1(2):82-8.

7. Kolar DR, Rodriguez DLM, Chams MM, Hoek HW. Epidemiology of eating disorders in Latin America: a systematic review and meta-analysis. Curr Opin Psychiatry. 2016;29(6):363-71.

8. Ministério da Saúde (BR), Secretaria de Vigilância em Saúde. VIGITEL Brasil 2014: vigilância de fatores de risco e proteção para doenças crônicas por inquérito telefônico [Internet]. Brasília: Ministério da Saúde; 2014 [acesso em 2016 Out 11]. Disponível em: 
http://bvsms.saude.gov.br/bvs/publicacoes/vigitel_ brasil_2014.pdf

9. Mertens F, Fillion M, Saint-Charles J, Mongeau P, Távora R, Passo CJS, et al. The role of strong-tie social networks in mediating food security of fish resources by a traditional riverine community in the Brazilian Amazon. Ecol Soc. 2015; 20(3):18-22.

10. Malta DC, Andrade SC, Claro RM, Bernal RTI, Monteiro CA. Evolução anual da prevalência de excesso de peso e obesidade em adultos nas capitais dos 26 estados brasileiros e no Distrito Federal entre 2006 e 2012. Rev Bras Epidemiol. 2014;17(Supl 1):267-76.

11. Oliveira BFA, Mourão DS, Gomes N, Costa JMC, Souza AV, Bastos WR, et al. Prevalência de hipertensão arterial em comunidades ribeirinhas do Rio Madeira, Amazônia Ocidental Brasileira. Cad Saúde Pública. 2013;29(8):1617-30.

12. Lino MZR, Muniz PT, Siqueira KS. Prevalência e fatores associados ao excesso de peso em adultos: inquérito populacional em Rio Branco, Acre, Brasil. Cad Saúde Pública. 2011;27(4):797-810.

13. Simoes EJ, Bouras A, Cortez-Escalante JJ, Malta DC, Porto DL, Mokdad AH, et al. A priority health index identifies the top six priority risk and related factors for non-communicable diseases in Brazilian cities. BMC Public Health. 2015;15(1):1

14. Apovian CM. The Obesity Epidemic: understanding the disease and the treatment. New Engl J Med. 2016;374(2):177-9.

15. Silva JC Neto, Aleixo NCR. Apropriação da natureza e processos erosivos na Região do Médio Solimões AM. Rev GeoUECE. 2014;3(40):151-76.

16. Instituto Brasileiro de Geografia e Estatística. Censo 2010: Cidades [acesso em 2016 Out 11]. Disponível em: http:/www.cidades.ibge.gov.br/painel/populacao. php?lang=\&codmun $=130420 \&$ search $=\% 7$ Ctefe

17. Instituto Brasileiro de Geografia e Estatística. Pesquisa Nacional de Saúde: percepção do estado de saúde, estilo de vida e doenças crônicas: 2013 [Internet]. Rio de Janeiro: IBGE; 2014. [acesso em 2016 Out 11]. Disponível em: http://www.pns.icict.fiocruz.br/arquivos/ Novos/arquivos/Novos/Qustionario\%20PNS.pdf

18. World Health Organization. Obesity: preventing and managing the global epidemic [Internet]. Geneva: WHO; 2000. (WHO Technical Report Series 894) [acesso em 2016 Out 11]. Disponível em: http:// www.who.int/nutrition/publications/obesity/WHO TRS 894/en/
19. James PA, Oparil S, Pharm BLC, Cushman WC, Dennison-Himmelfarb C, Handler J, et al. 2014 Evidence-Based Guideline for the Management of High Blood Pressure in Adults. Report From the Panel Members Appointed to the Eighth Joint National Committee (JNC 8). JAMA. 2014;311(5):507-20.

20. Sociedade Brasileira de Diabetes. Diretrizes da Sociedade Brasileira de Diabetes (2015-2016) [acesso em 2016 Out 11]. Disponível em: http://www. diabetes.org.br/sbdonline/images/docs/DIRETRIZESSBD-2015-2016.pdf

21. Brevidelli MM, Coutinho RMC, Costa LFV, Costa LC. Prevalência e fatores associados ao sobrepeso e obesidade entre adolescentes de uma escola pública. Rev Bras Promoç Saúde. 2015;28(3):379-86.

22. Stevens GA, Singh GM, Lu Y, Danaei G, Lin JK, Finucane MM, et al. Global burden of metabolic risk factors of chronic diseases collaborating group (body mass index) - national, regional, and global trends in adult overweight and obesity prevalences. Popul Health Metr. 2012;10(1):10-22.

23. Kramer CK, Zinman B, Retnakaran R.Are metabolically healthy overweight and obesity benign conditions?: A systematic review and meta-analysis. Ann Intern Med. 2013;159(11):758-69.

24. Jaime PC, Stopa AR, Oliveira TP, Vieira ML, Szwarcwald CL, Malta DC. Prevalência e distribuição sociodemográfica de marcadores de alimentação saudável, Pesquisa Nacional de Saúde, Brasil 2013. Epidemiol Serv Saúde. 2015;24(2):267-76.

25. Bezerra IN, Cavalcante JB, Moreira TMV, Mota CC, Sicheiri R. Alimentação fora de casa e excesso de peso: uma análise dos mecanismos explicativos. Rev Bras Promoç Saúde. 2016;29(3):455-61.

26. International Food Policy Research Institute. Global nutrition report 2014: actions and accountability to accelerate the world's progress on nutrition [Internet]. Washington: IFPRI; 2014 [acesso em 2016 Out 11]. Disponível em: http://www.ifpri.org/sites/default/files/ publications/gnr14.pdf

27. Tavares RS, Silva DMGV, Sasso GTM, Padilha MICS, Santos CRM. Fatores de riscos cardiovasculares: estudo com pessoas hipertensas, de um bairro popular na região amazônica. Ciênc Cuid Saúde. 2014;13(1):411.

28. Popkin BM, Slining MM. New dynamics in global obesity facing low- and middle-income countries. Obes Rev. 2013;14(Suppl 2):11-20. 
29. Carlucci SEM, Gouvêa JAG, Oliveira AP, Silva JD, Cassiano ACM, Bennemann RM. Obesidade e sedentarismo: fatores de risco para doença cardiovascular. Com Ciências Saúde. 2013;24(4):37584.
Endereço do primeiro autor:

Jéssica Yelle Ferreira Cordeiro

Universidade do Estado do Amazonas - UEA

Departamento de Ciências Biológicas, Centro de Estudos Superiores de Tefé

Estrada do Bexiga, 1085

Bairro: Juruá

CEP: 69552-315 - Tefé - AM - Brasil

E-mail: jecika_yelle@hotmail.com

Endereço para correspondência:

Sílvia Regina Sampaio Freitas

Universidade do Estado do Amazonas - UEA

Departamento de Ciências Biológicas, Centro de Estudos Superiores de Tefé

Estrada do Bexiga, 1085

Bairro: Juruá

CEP: 69552-315 - Tefé - Amazonas - Brasil

E-mail: silvia.sampaio.freitas@hotmail.com 